\title{
Early Aspects at ICSE 2007: Workshop on Aspect-Oriented Requirements Engineering and Architecture Design
}

\author{
Ruzanna Chitchyan ${ }^{1}$, Awais Rashid ${ }^{1}$, Ana Moreira ${ }^{2}$, João Araújo², Paul Clements ${ }^{3}$, Elisa \\ Baniassad $^{4}$, Bedir Tekinerdogan ${ }^{5}$ \\ ${ }^{1}$ Lancaster University, UK; ${ }^{2}$ New University of Lisbon, Portugal; ${ }^{3}$ Software Engineering \\ Institute, USA; ${ }^{4}$ Chinese University of Hong Kong, Hong Kong; ${ }^{4}$ University of Twente, The \\ Netherlands. \\ \{rouza|marash\}@comp.lancs.ac.uk; \{amm|ja\}@di.fct.unl.pt; clements@sei.cmu.edu; \\ clements@sei.cmu.edu; bedir@cs.utwente.nl
}

\begin{abstract}
The "Early Aspects@ @ ICSE'07" is the 11 $1^{\text {th }}$ workshop in the series of Early Aspects workshops [1] which focuses on aspect identification during the requirements engineering and architecture derivation activities. The specific aim of the present workshop is twofold: (a) to initiate creation of an Early Aspects application demonstration and comparisons benchmark; and (b) to solicit submission of new research.
\end{abstract}

\section{Early Aspects at ICSE'07: Aims and Objectives}

Early aspects are crosscutting concerns that exist in the early life cycle phases of software development, including the requirements analysis, domain analysis and architecture design activities. Early aspects aim to localize requirements and architectural concerns whose influence would otherwise tend to be scattered over multiple early life cycle modules, leading to serious maintenance problems. Since these early development phases produce the early design decisions that have the most profound impact on the whole system, dealing with aspects at these early life cycle phases is critical. This workshop builds on the work started with the previous Early Aspects (EA) workshops to strengthen cross-fertilization of ideas in requirements engineering, domain engineering, software architecture design and aspect-oriented software development. The specific aim of the present workshop is twofold:

1. to initiate creation of an EA application demonstration and comparisons benchmark; and

2. to solicit submission of new research.

While promotion and discussion of new research is an established and continuing goal of this workshop series, the building of the EA approaches comparison benchmark is a new one and is the most specific objective of this workshop. In order to initiate creation of an Early Aspects' application demonstration and comparisons benchmark the workshop has invited submissions that demonstrate how the submitter's EA approach can be applied to a suggested benchmark case study. The selected case study is that of the Health Watcher [2] system presently used in Brazil for public health monitoring and complaint reporting. It is hoped that using a consistent case study for presenting the current early aspects approaches will facilitate their comparison as well as help identify areas that are not well addressed in the present work, thus setting out further research directions.

To facilitate comparison of the approaches, the workshop has suggested a structure for the benchmark case study papers. These papers will be structured in accordance with a number of required and optional sections. The required sections focus on presentation of the approach. The optional sections focus on such important issues, as composition support, trade-off point identification, trade-off resolution, and tool support. These sections are optional only because not every approach will cover all these issues. To facilitate comparison, it is agreed that if an optional section is omitted, the readers should assume that the topic of the section is not supported by the presented approach.

The accepted comparison papers will form the basis of the EA approaches comparison repository. We indent to maintain and extend this repository through the future workshops for the benefit of the whole of the EA community.

\section{Workshop Activities}

The workshop is intended to be an interactive event with a focus on making tangible progress. During the 
morning three or four short presentations of the accepted papers will be given. Presentations will be selected on such topics that are most likely to generate fruitful discussions during the rest of the day. The bulk of the workshop will be reserved for group discussions and overall conclusions on the topics raised by the presentations as well as elicited by the reviewers and participants from the other accepted papers.

The submitted benchmark papers will be used as basis for a panel discussion entitled "Comparing Early Aspects Approaches" at the workshop.

After the workshop the participants will be invited to collaborate on preparing the workshop report and augmenting the EA landscape report [3] which is the result of the effort of the previous EA workshops. The benchmark paper submissions will form contribution towards the Early Aspects Benchmark Repository.

\section{Summary}

Aspect-Oriented Software Development (AOSD) techniques provide systematic means for the identification, modularization, representation and composition of crosscutting concerns such as security, mobility and real-time constraints. The Early Aspects Initiative focuses on managing crosscutting properties at the early development stages of requirements engineering and architecture design. The EA workshop series [1] have been the main means of the EA community for disseminating, discussing, and crossfertilizing work in this new research area. The present workshop keeps to these same objectives, while also setting an additional aim of building a new recourse for the EA community - the Early Aspects approaches comparison benchmark and repository.

\section{Acknowledgements}

The organizers would like to thank the workshop program committee and a number of additional reviewers for their help.

Program Committee:

- Vander Alves, University of Lancaster, UK

- Thais Batista, Universidade Federal do Rio Grande do Norte, Brazil

- Jaelson Castro, Universidade Federal de Pernambuco, Brazil
- Anthony Finkelstein, University College London, UK

- Alessandro Garcia, University of Lancaster, UK

- Gerri Gerog, Colorado State University, USA

- Michael Jackson, The Open University, UK

- John McGregor, Clemson University, USA

- Paulo Merson, Software Engineering Institute, USA

- Bashar Nuseibeh, The Open University, UK

- Mónica Pinto, Universidad de Málaga, Spain

- Jon Whittle, George Mason University, USA Additional Reviewers:

- Eduardo Figueiredo, University of Lancaster, UK

- Praveen Jayaraman, George Mason University, USA

- Robin Laney, The Open University, UK

- Claudio Sant'Anna, University of Lancaster, UK

- $\quad$ Lyrene Silva, PUC-Rio, Brazil

- Nathan Weston, University of Lancaster, UK

- Yijun Yu, The Open University, UK

We also thank Sérgio Agostinho from the New University of Lisbon, Portugal for help with the web support.

The Early Aspects at ICSE'07 workshop is sponsored by the AOSD-Europe Network of Excellence, http://www.aosd-europe.net/, (EC Grant: IST-2-004349).

\section{References}

[1] Early Aspects.net, web site for the "Early Aspects: Aspect-Oriented Requirements Engineering and Architecture Design", http://early-aspects.net/ .

[2] S. Soares, E. Laureano, P. Borba, "Implementing Distribution and Persistence Aspects with AspectJ", 17th International Conference on Object-Oriented Programming, Systems, Languages, and Applications (OOPSLA' 02), ACM Press, November 2002, pp. 174-190.

[3] "Early Aspects: the Current Landscape" resultant report of the EA workshop series, available at http://trese.cs.utwente.nl/early-aspects-

AOSD2005/Papers/EarlyAspects-LandscapePaper-

FirstDraft-2005.pdf 